\title{
Investigation into the numerical model behaviour of Belleville washers in cold roll forming
}

\author{
Kwun Sing Tsang ${ }^{1, \mathrm{a}}$, William Ion ${ }^{1, \mathrm{~b}}$, and Martin English ${ }^{2, \mathrm{c}}$ \\ ${ }^{1}$ Department of Design, Manufacture and Engineering Management, University of Strathclyde, \\ 75 Montrose Street, Glasgow G1 1XJ, UK \\ ${ }^{2}$ Hadley Group, Downing Street, Smethwick, Birmingham, West Midlands B66 2PA, UK \\ akwun.tsang@strath.ac.uk, bw.j.ion@strath.ac.uk, cmartin.english@hadleygroup.com
}

Keywords: Cold roll forming, numerical simulation, Belleville washers.

\begin{abstract}
With the steady increase in demand for roll formed products, due to their application in fields such as automotive, construction, architecture, etc., roll forming companies are challenged with rising customer demands. Companies are struggling to bring a product through from design to manufacture at improved rates, whilst achieving tighter tolerances. The roll forming process may also induce undesirable forming defects such as twist, distortion and straightness problems. Over the years, numerical simulations have been deployed to assist with the geometric validation of these effects. For the purpose of this paper, an investigation shall focus on an individual parameter within the finite element analysis of the cold roll forming process, i.e. the 'unknown' spring effect acting on the top roll during the forming process due to the implementation of Belleville washers in the pillar set up. This parameter is assessed over a range of applied loads and compared with a standard friction model with fixed top and bottom rolls. The longitudinal strains on the final profile, the geometric accuracy and thickness variation are observed and presented in this paper. Reasoning is also provided to explain why the final profile bows in the vertical direction.
\end{abstract}

\section{Introduction}

Finite element analysis software packages have been developed for over forty to fifty years now and have been deployed to assist with the geometric validation of the forming behaviour of roll formed products. This forming process can produce a wide variety of profiles, which in the past the associated tooling design has been mainly achieved through a combination of an empirical approach and heuristic process [1,2]. As the variety and complexity of roll formed products increase with the additional requirement to satisfy relentless tolerances, more steps are required to meet this demand, i.e. extended product design development, consideration of alternate forming strategies, additional forming stages, etc. As the roll forming design moves away from this empirical approach to adopt a numerical simulation methodology, there is an associated time and cost saving due to the reduction of the commissioning phase, the cut down of trial and error parts and also little demand for re-making of tooling. This brings the product available to market at a significantly quicker rate. Other main benefits include the ability to virtually investigate changes in specific parameters without the risk of expensive tooling and to apply a quality control before a component is even made.

The industry partner of this project, Hadley Group, Birmingham, UK, implement Belleville washers into the roll tool configuration as shown in Figure 1. The purpose of these washers is to apply pressure to the material strip during forming in order to drive material through each forming station. They are also used to adjust the gap between the top and bottom forming rolls, since the incoming material gauge can vary by the nominal tolerance values from coil to coil.

The objective of this paper is to carry out an investigation focusing on an individual parameter within the finite element analysis (FEA) of the cold roll forming process, i.e. the 'unknown' spring effect acting on the top roll during the forming process due to the implementation of Belleville washers in the pillar set up. 


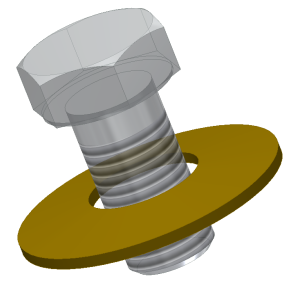

(a) Belleville washer

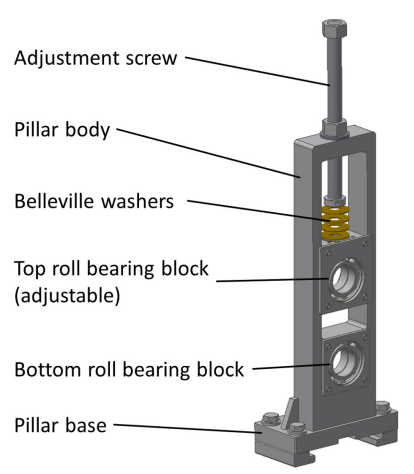

(b) Schematic

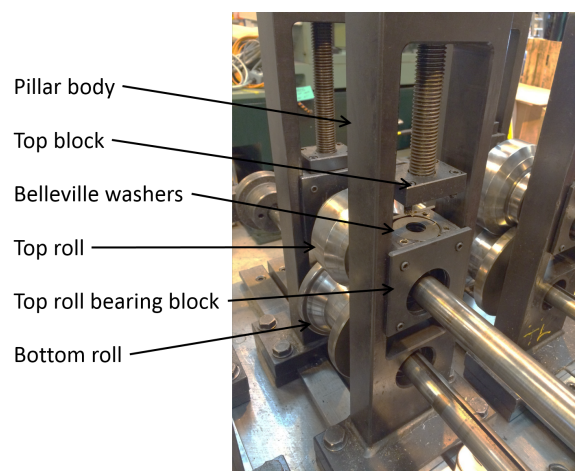

(c) Image

Fig. 1: Roll forming pillar configuration

\section{Roll Forming}

Cold roll forming is a manufacturing process which dates back many hundreds of years. It is comprised of using sheet metal to form a desired component with a uniform cross section. This process is also referred to as contour roll forming and is ideal for producing components that have a constant cross sectional profile with long lengths and when large quantities are required. Hence, roll formed parts are advantageous due to the high productivity at a low cost. The raw material consists of flat metal strip which is fed through a number of consecutive forming passes. Each forming pass is comprised of a set of corresponding roll tools and through each pass the raw material is incrementally formed until the final profile is achieved as shown in Figure 2. There are numerous reasons which might prohibit the forming of accurate profiles. To name only a few principles that the designer must consider for a successfully formed profile, as described by English include determining the sequence of bends (flower plan), the strip width required, the effects acting on the strip material by the roll tools and the effects of residual stresses during the design of a new product [3]. Hence why successful roll forming has been considered an art in which sectional and longitudinal defects are avoided whilst forming a desired cross section from a flat metal strip.

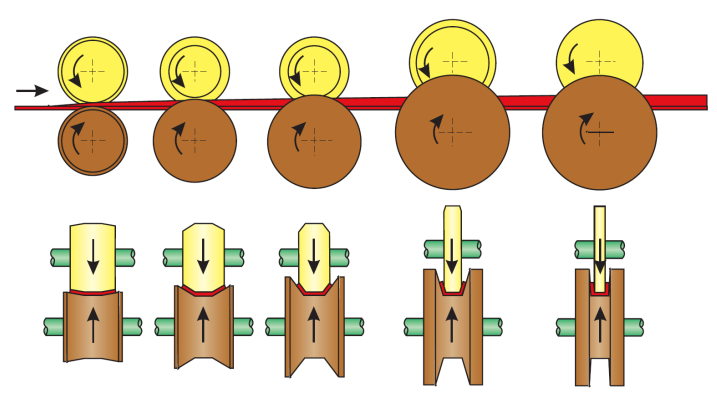

(a) Schematic [4]

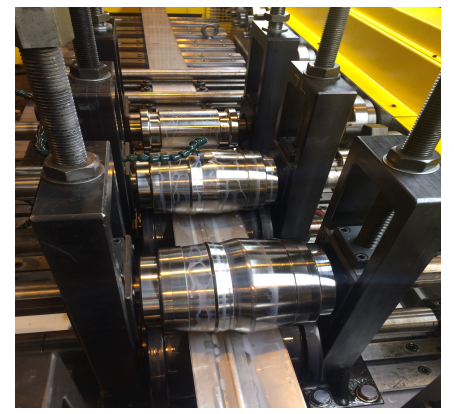

(b) Image

Fig. 2: Roll forming process

Within Hadley Group, Birmingham, the largest independent manufacturer of cold rolled metal sections and profiles in the UK, there is a unique assembly for each forming pass which includes the implementation of Belleville washers when compared to the conventional set up. The top rolls in the roll forming process are subjected to an unknown vertical loading condition due to the use of these washers in the side pillars as shown in Figure 1, which acts as a spring. The main purpose of these washers is to apply pressure to the strip material during roll forming in order to drive the material through each forming station. In addition to this, adjusting the pressure on the top roll can address 
some of the forming defects mentioned previously. In particular, twist is a result of when an excess of forming pressure is applied to the forming material [5]. Should the pressure be increased then thinning may also occur. Consequently, this elongation of the strip material may result in waving. By including these washers, the operator possesses greater control over the magnitude of pressure applied. This is due to the range of the washers' deflection which is directly proportional to the applied pressure on the profile. Should the washers be removed then the pressure applied ranges from no pressure at all to a maximum with minimum turns of the adjustment screw as shown in Figure 1. Hence, with no washers it is very difficult to control the magnitude of pressure applied.

Another advantage of implementing these Belleville washers is due to the nature of their size. Only a small limited amount of space is required and the option of stacking these in parallel or series is available to the designer as shown in Figure 3.

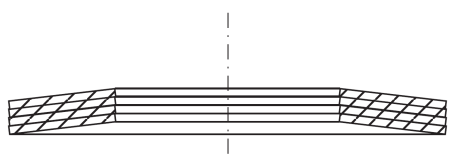

(a) Parallel

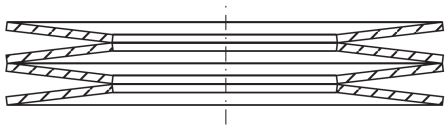

(b) Series

Fig. 3: Example of stacked Belleville washers [6]

It has been noted by Pedersen and Pedersen [6] that using these washers in parallel introduces losses primarily due to friction between each other. This has been supported by work published by Curti and Montanini [7]. As these washers are implemented in series, these losses can be neglected.

Numerical Analysis. With the advances in the computational power and technology, finite element analysis has been widely implemented to predict the forming behaviour of sheet metal for many processes to include cold roll forming. There is still a challenge in acquiring precise and reliable results, however, this is substantially influenced by the user's knowledge and competency. Note that numerical simulations are only to be used as a guide, naturally these tools do not provide a solution with $100 \%$ accuracy due to assumptions applied.

Early work conducted by Duggal et al. [2] compares simulation analysis with experimental for the forming of simple $\mathrm{U}, \mathrm{V}$ and $\mathrm{C}$ channels. This work similar to many others uses simple profiles to minimise the amount of variables present in the process. This should provide a true insight into the influence of the parameters being tested.

Research surrounding the application of computational and experimental techniques to metal deformation in roll forming was provided by Senanayake et al. [8] where the main objective of this work was to gain a better understanding of how material behaves during the roll forming process. A trapezoidal section was investigated using a commercial numerical simulation package - MSC MARC/MENTAT.

More recently Bui and Ponthot [9] compares the longitudinal strains and trajectories of the displacement between both experimental and numerical approaches. It was noted that in this work perfectly rigid die roll was applied based on the assumption that the dies do not deform when subjected to the forming process. The author applies the suggested Coulomb friction model by Hong et al. [1], with $\mu=0.2$ as the coefficient of friction.

Should simulation be required to be simplified further, without compromising the results, Sheu [10] successfully altered the boundary conditions whilst achieving this. As opposed to the roll tools defined as stationary with the strip material moving towards them at a given speed, the strip is modelled as static and the roll tools driven over the material. 


\section{Numerical Simulation model set-up}

MSC MARC MENTAT 2014.0.0, developed by MSC Software is the finite element analysis software to be used for this investigation. MENTAT is used for the pre and post-processing of the simulation, while the analysis is computed by MSC MARC. AutoCAD 2015 and COPRA®FEA RF are the software used to design the tool and profile geometry.

A trapezoidal profile was selected for investigation in this current study. The profile was subjected to three forming passes bringing a flat strip material to a bend of $55^{\circ}$ on both legs as shown in Figure 4 and Figure 5. The profile is not formed past $55^{\circ}$ as side rolls would have to be introduced to comply with best practice. Should side rolls be implemented, there would naturally be an increase of process variables which may disguise the true effect of top roll pressure variation on the formed profile. As the profile is symmetric only half the profile is modelled to reduce the size of the model. Ultimately, this will reduce the total element count and the computational power required to complete the simulations.

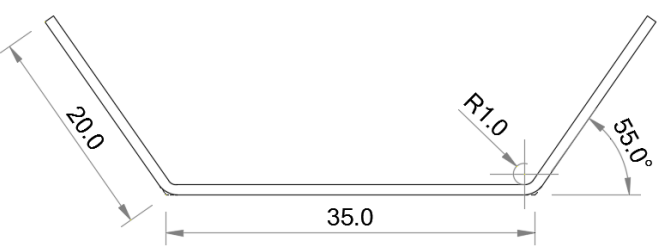

(a)

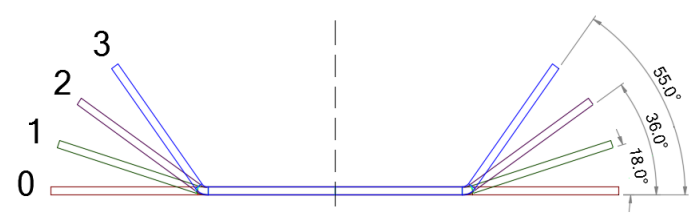

(b)

Fig. 4: (a) Final profile \& (b) Flower plan / Bending sequence



Fig. 5: Numerical simulation - model set up

The roll forming process was simulated under a number of conditions, the first consisting of a standard friction model where the sheet metal is formed through rotating rolls fixed in position; and the remaining simulations consisting of the top roll in the third forming pass applying a pressure onto the sheet metal whilst being subjected to a vertical displacement. The pressure applied was varied to determine the influence of this parameter on the final profile.

The strip material used in this investigation consists of galvanised mild steel and for this paper was considered as an isotropic material. This assumption was applied in order to simplify the simulation. The material in question has a Young's modulus of $205 \mathrm{GPa}$, yield strength of $140 \mathrm{MPa}$ and Poisson's ratio of 0.3 as specified in Hadley Group's material library [11]. It is assumed that the thermal properties are negligible. This strip material was assigned as a deformable body. It is to be noted that for any contact analysis, at least one deformable body is required. Element number 7, a 8-node hexahedral element type was selected to model the three dimensional solid of the strip. This element type has eight integration points and is preferred when a contact analysis is conducted [12].

The material for the roll tools in this forming process is typically through hardened D2 tool steel. It is assumed that there will be little deformation to the tool material during this process due to the high stiffness of the material, and thus the material will not plastically deform. Based on this assumption 
the surfaces of the rolls are defined as rigid and therefore will not deflect at all. No material properties are required.

Note that there are a number of ways in which rigid bodies can be controlled. In particular, rigid bodies can be controlled via load or position. The top rolls were controlled by load while the bottom rolls were controlled by position. This was applied to all three sets of rolls. A standard friction model was simulated, with the strip driven through the rotating rolls due to the contact between the bodies. The coefficient of friction, $\mu$ was 0.21 for this investigation. The bottom rolls were driven and fixed in their original position. The top rolls were not driven and may rotate freely. Note that for a non-friction model, where the rolls do not rotate and are driven over the material, both rolls would be controlled via position. Although Bui and Ponthot described friction as negligible, a friction model is used due to the current computational capabilities which means simplification of the model is not essential. With this model, the behaviour of the top roll can also be configured with much more ease. In this study, it was necessary to release the top roll constraint in the vertical y-direction when a force is applied upon it.

The forces applied to the top roll ranged from $2000 \mathrm{~N}$ to $7000 \mathrm{~N}$ and increase in increments of 1000 N. For the purpose of this investigation it was assumed that these forces remain constant during the forming process. These values were applied due to the data provided in Figure 6 which presents the load vs. displacement properties of the Belleville washers in question. These washers were sourced from Belleville Springs Ltd, Redditch, UK and are specified as D5020425. For four disk springs stacked in series, the total force required for maximum deflection is equal to the force required of a single spring. However, the total deflection is equal to four times the deflection of a single disk spring. The maximum force applied was set to $7000 \mathrm{~N}$ in this investigation in order to avoid exceeding $75 \%$ of the maximum deflection, which would result in significant force increase and stress characteristics [13].

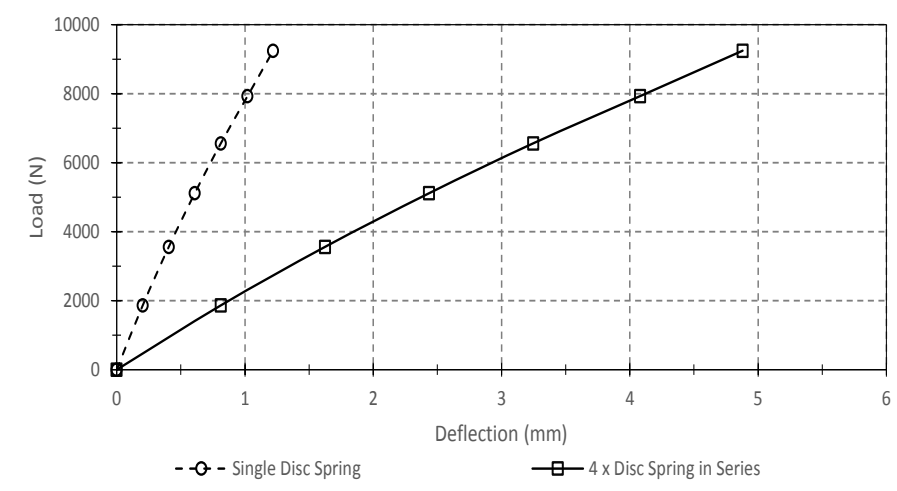

Fig. 6: Force vs. displacement data - washers in series

It is worth noting that the strip guide does not subject the material to any deformation as it consists of two flat rolls. However, it is an important component in the process as it applies a constraint on the material before it enters the first pass [14].

\section{Results and Discussion}

Upon completing the numerical simulations as described in the previous sections, the final profiles were analysed to determine the following points: geometric accuracy; final bend angle; and the thickness variation along the length of the strip. Before these points are discussed, the validation of the simulation model was reviewed.

Validation of Numerical Simulation. The numerical simulations carried out in this investigation were validated on the basis of the geometric correlation between numerical and experimental results 
for a standard friction model. Physical samples were obtained through the author's previous work, in which a similar trapezoidal profile was analysed [15]. The geometric aspects considered consisted of the straight element lengths of the profile $(a, b)$ the material gauge (c) and the final leg angle of bend (d) as shown in Figure 7. Table 1 presents the recorded measurements of elements a - $d$ for both the experimental and numerical simulations. It can be seen that the discrepancies are within $1 \%$, with the exception of the material gauge just under $5 \%$.

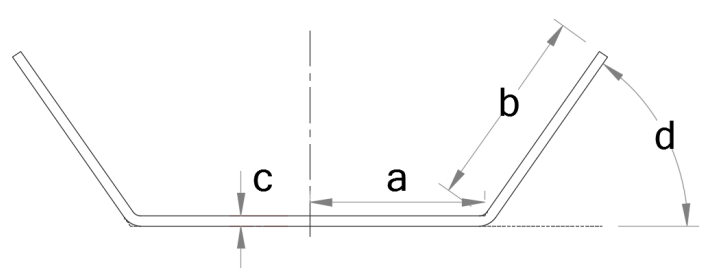

Fig. 7: Profile elements for validation

Table 1: Model validation - numerical vs. experimental data

\begin{tabular}{cccc}
\hline Element & Experimental & Numerical & Variation (\%) \\
\hline $\mathrm{a}$ & $17.769 \mathrm{~mm}$ & $17.661 \mathrm{~mm}$ & -0.608 \\
$\mathrm{~b}$ & $17.343 \mathrm{~mm}$ & $17.380 \mathrm{~mm}$ & +0.213 \\
$\mathrm{c}$ & $1.432 \mathrm{~mm}$ & $1.499 \mathrm{~mm}$ & +4.679 \\
$\mathrm{~d}$ & $54.546^{\circ}$ & $55.05^{\circ}$ & +0.933 \\
\hline
\end{tabular}

The successful validation of this model then progressed the investigation to subjecting the top roll in the third pass with variable pressures as previously described.

It is to be noted that while in this paper the assumption is made in that the simulation models are validated on the basis of geometry, there are other parameters worth considering. These validations may be conducted using strain measurement techniques such as Mylar tape or optical strain measurement, as well as hardness and micro-hardness mapping can be considered.

Geometric Accuracy. It can be observed that the profile leans down in the vertical direction as it exits the final forming pass. This behaviour is to be expected due to a number of reasons. The main reason is due to a larger bottom roll diameter forming the flange of the profile which results in an inconsistency of longitudinal strains across the profile. It is shown in the work of Paralikas et. al how the flange travels a greater distance than the web to support this point [16]. The longitudinal strain along the flange and web in the rolling direction for the original standard friction model simulation is presented in Figure 8(a). It is clear from this graph that the flange is mainly under tension, and the web under compression. This results in the elongation of the flange which becomes longer than the web in length, hence, the vertical bow behaviour occurs. A common method of addressing this issue is to subject the profile to an additional two forming passes which are similar to the final forming pass. However, these final two passes, known as lifter passes are to have a larger bottom roll diameter to counter the issue of the profile running vertically down.

The magnitude in which this vertical displacement occurs is measured and presented in Figure 9 for each simulation set up. There is a clear trend illustrating that as the load applied is increased, the vertical displacement in the negative direction decreases. The profile is shown to bow up in the vertical direction when a load of $7000 \mathrm{~N}$ is applied. The reason for this is a result of the thinning effect along the web. As the force is increased, the web of the profile is stretched longitudinally. Once this longitudinal strain exceeds the longitudinal strain along the flange, the profile will travel up in a vertical direction as opposed to down. This difference of strains is clearly illustrated in Figure 8(b). 


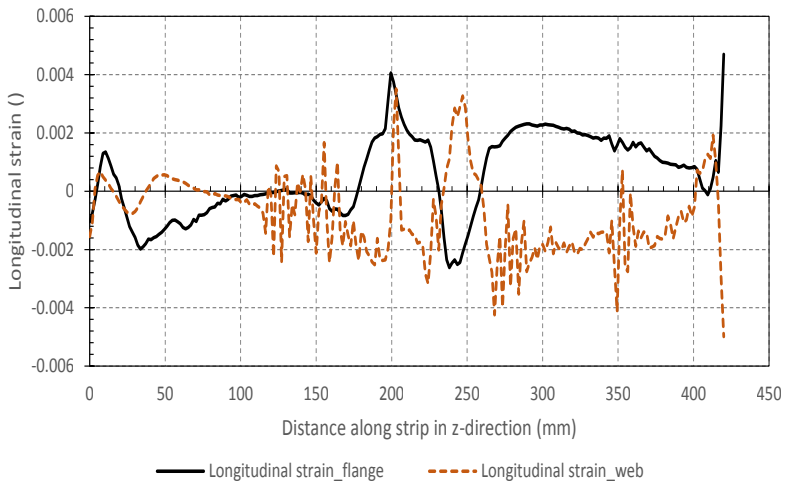

(a)

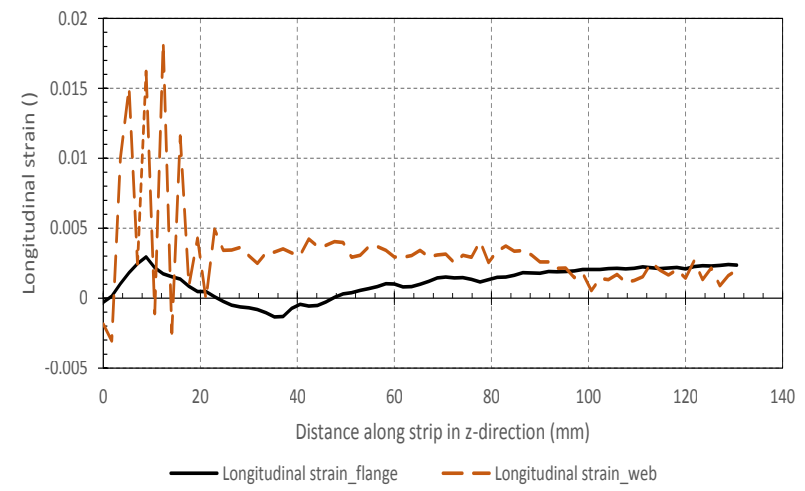

(b)

Fig. 8: Longitudinal strain along web \& flange (a) standard friction model \& (b) $7000 \mathrm{~N}$ applied load

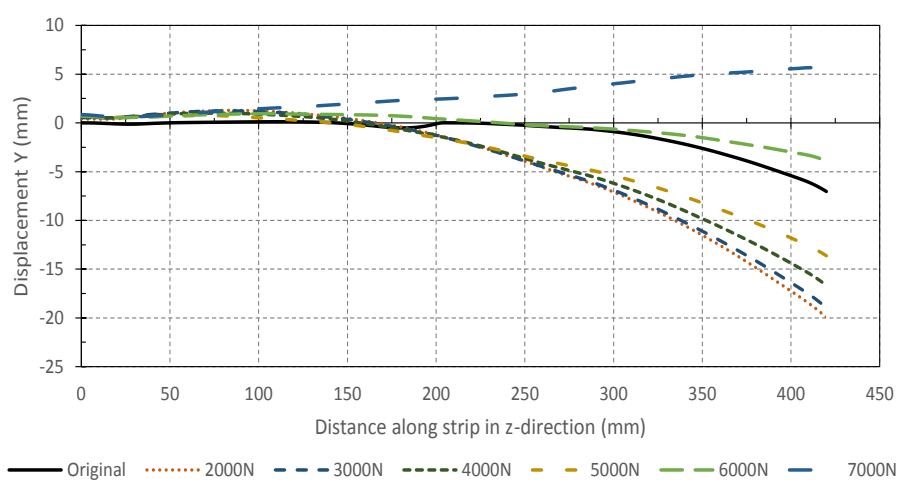

Fig. 9: Vertical displacement along web

Final Bend Angle. The final bend angles of each profile has been recorded and presented in Table 2 . A cross section of the profile was separated $150 \mathrm{~mm}$ from the back edge in order to measure this angle. The front section of the profile was discarded in order to neglect the forming defects which occur when the strip first comes into contact with each forming pass, i.e. buckling. These angles obtained through numerical simulation are compared against that measured for the original standard friction simulation where no vertical pressure is applied to the top roll. It is clear that there is little variance between the final bend angle when different forces are applied with the variation ranging from $-0.24 \%$ to $0.36 \%$.

Table 2: Final bend angles

\begin{tabular}{ccc}
\hline Load applied $(\mathrm{N})$ & Final bend angle $\left(^{\circ}\right)$ & Variation $(\%)$ \\
\hline 0 & 54.83 & 0 \\
2000 & 54.70 & -0.24 \\
3000 & 54.82 & -0.02 \\
4000 & 54.92 & 0.16 \\
5000 & 54.98 & 0.27 \\
6000 & 55.03 & 0.36 \\
7000 & 54.91 & 0.15 \\
\hline
\end{tabular}

The reason as to why there is a minor reduction in the measured final bend angle to that shown in the flower plan, i.e. $55^{\circ}$, is due to a number of possible factors. The main factor may be due to the influence of springback. Springback occurs at the end of a forming process and is an elastically-driven change of 
the parts shape upon unloading. This is also known as elastic recovery and results in geometric change which is an undesired effect as this decreases geometric accuracy. The degree in which a material will unbend depends on the material characteristics of the strip, i.e. yield, elastic modulus, etc. In order to overcome this, material is usually bent more than what is required, and upon releasing the load, springback will bring the material to the desired bend angle. The angle in which the material is over formed can be determined by the designer's intuition and experience, through analytical methods or via software such as Deformation Technology Module. It can be noted that springback is significantly reduced as the tensile stress acting on the material approaches the materials yield stress [17].

Thickness Variation. The thickness variation along the longitudinal direction of the strip is presented in Figure 10. This data represents the thickness strain for the following three simulation configurations: the original standard friction model simulation where no force is applied to the top roll; and when a $2000 \mathrm{~N}$ and $7000 \mathrm{~N}$ load is applied.

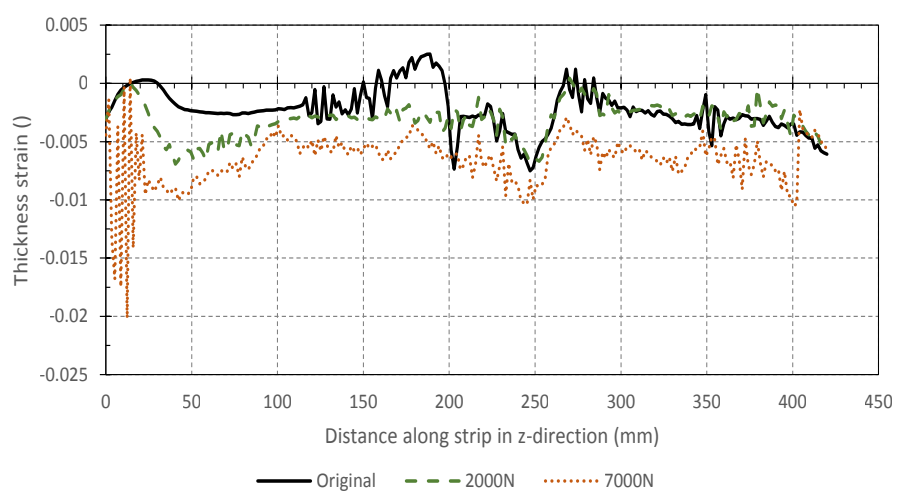

Fig. 10: Thickness variation along web

This data is collected to illustrate that some thinning occurs in each of the simulation set ups. However, it is clear that as the applied load is increased, the magnitude of thinning is also increased. This must be taken into consideration for new tooling designs as the designer must satisfy tolerances associated with the profile. Therefore, the forming loads should be reduced or a thicker material should be specified to meet the final profile thickness required if thinning cannot be avoided.

\section{Conclusion}

The numerical simulation of a roll forming process has been presented in this current investigation. The final profile in this study takes the shape of a trapezoidal section, with the flanges formed to an angle of $55^{\circ}$. Three forming passes were modelled with the strip material consisting of a standard galvanised mild steel. The modelling process has been described to include the behaviour of the top roll when a vertical force is applied and how this effects the final profile. This force is applied to reflect the behaviour of Belleville washers in the current pillar configuration in Hadley Group, UK. The simulations are compared against a standard friction model in which both the top and bottom roll are fixed in position, with the bottom roll driven. Specific results obtained through this study include the longitudinal strain along the rolling direction for both the web and flange element; the magnitude of bowing in the vertical direction and the thickness variation along the profile. Ultimately, these results explain how the geometric accuracy is influenced by the force applied in the forming process. The main relationships between force and geometric accuracy are outlined below:

- Increase in force $\rightarrow$ reduction of material thickness;

- Increase in force $\rightarrow$ increase of material deflecting up in vertical direction; and

- Change in force $\rightarrow$ minimum influence on final bend angle. 
Future work may include further development of the numerical model for the improved prediction of final profile geometry. A non-linear load may be applied to represent the Belleville washers behaviour as a function of deflection. In addition to this, analysis of the springback characteristics may also be conducted. Furthermore, the additional validation methods involving strain measurement and hardness mapping may be considered to further verify the numerical model. Note that there are numerous variables within the roll forming process, each should be investigated individually before compiling a number of these together to model a robust simulation.

\section{References}

[1] S. Hong, S. Lee, N. Kim, A parametric study on forming length in roll forming, J. Mater. Process. Technol. 113 (2001) 774-778.

[2] N. Duggal, M.A. Ahmetoglu, G.L. Kinzel, T. Altan, Computer aided simulation of cold roll forming - a computer program for simple section profiles, J. Mater. Process. Technol. 59 (1996) 41-48.

[3] M.A. English, Minimisation of waste through the development of a knowledge based engineering system for the cold roll forming process, PhD thesis (2008).

[4] Schular, Metal forming handbook, Springer Science \& Business Media. (1998).

[5] ASM Handbook, Volume 14 - Forming and forging, ASM Int. 14 (1996) 1376-1400.

[6] N.L. Pedersen, P. Pedersen, Stiffness and design for strength of trapezoidal Belleville springs, J. Strain Anal. Eng. Des. 46 (2011) 825-836.

[7] G. Curti, R. Montanini, On the influence of friction in the calculation of conical disk springs, J. Mech. Design. 121 (1999) 622-627.

[8] R. Senanayake, I. Cole, S. Thiruvarudchelvan, The application of computational and experimental techniques to metal deformation in cold roll forming, J. Mater. Process. Technol. 45 (1994) 155-160.

[9] Q.V. Bui, J.P. Ponthot, Numerical simulation of cold roll-forming processes, J. Mater. Process. Technol. 202 (2008) 275-282.

[10] J.J. Sheu, in: Proceedings of the 8th Int. Conference on Numerical Methods in Industrial Forming Processes, Columbus, Ohio, AIP Publishing (2004).

[11] Hadley Group, Material properties summary chart. Unpublished internal document (2015).

[12] MARC®2014, Volume b: Element library, (2014) 71-72.

[13] Information on http://www.bellevillesprings.com/index.html

[14] J. Paralikas, K. Salonitis, G. Chryssolouris, Investigation of the effects of main roll-forming process parameters on quality for a V-section profile from AHSS, Int. J. Adv. Manuf. Technol. 44 (2009) 223-237.

[15] K.S. Tsang, Validation of experimental and numerical model behaviour of Belleville washers in cold roll forming, MSc dissertation, University of Strathclyde (2015).

[16] J. Paralikas, K. Salonitis, G. Chryssolouris, Optimization of roll forming process parameters - a semi-empirical appraoch, Int. J. Adv. Manuf. Technol. 47 (2010) 1041-1052.

[17] W.D. Carden, L.M. Geng, D.K. Matlock, R.H. Wagoner, Measurement of springback, Int. J. Mech. Sci. 44 (2002) 79-101. 